\title{
Peptide receptor radionuclide therapy plus somatostatin analogues for a neuroendocrine tumour combined and maintenance treatment
}

\author{
Salvatore Antonio Pignata ${ }^{1}\left[\right.$ Andrea Ferrantelli $^{2}{ }^{\mathbb{D}} \cdot$ Sergio Baldari $^{1}$
}

Received: 4 July 2019 / Accepted: 13 August 2019 / Published online: 2 September 2019

(c) Italian Association of Nuclear Medicine and Molecular Imaging 2019

Neuroendocrine tumours (NETs) constitute a diverse group of cancers characterized by heterogeneous biological hallmarks and variable behaviour, from slow-growing and indolent tumours to aggressive and rapidly fatal cancers [1].

Somatostatin analogues (SSAs) provide the first line of systemic treatment of the gastro-entero-pancreatic-NETs (GEP-NETs), through the expression of somatostatin receptors (SSTRs) by NETs cells that bind the SSAs. The SSAs control the symptoms related to hormone hypersecretion in symptomatic GEP-NETs patients, inhibiting tumour angiogenesis and consequently its growth. This improves both progression-free survival (PFS) and overall survival (OS) [2].

Another option for the treatment of advanced, metastatic, well-differentiated and SSTRs-positive NETs is given by the peptide receptor radionuclide therapy (PRRT), which has been successfully applied since the 1990s [1]. The treatment for patients with progressive metastatic NETs, usually consisting of 4 cycles of $7.4 \mathrm{GBq}(200 \mathrm{mCi})$ of $\left[{ }^{177} \mathrm{Lu}\right]$ DOTApeptide at 8 weekly intervals, has proven to be safe, effective and with low side-effects [1]. A number of studies have shown a significant clinical benefit in NETs patients following PRRT. Brabander et al. confirmed a promising therapeutic outcome in 443 metastatic NETs' patients that expressed SSTRs [3]. The overall efficacy data showed good response rates, with, respectively, a PFS and OS of 29 and 63 months (64 months of median follow-up time and range 58-70 months).

Salvatore Antonio Pignata

spignata@unime.it

1 Nuclear Medicine Unit, Department of Biomedical and Dental Sciences and Morphofunctional Imaging, University of Messina, Via Consolare Valeria nr.1, 98125 Messina, Italy

2 Department of Civil Engineering and Architecture, Tallinn University of Technology, Ehitajate tee 5, 19086 Tallinn, Estonia
Until now, however, the data on OS and PFS have been established only for either SSAs or PRRT treatment, while their synergistic effect has been highlighted only recently. In 2014, Ezzidin and colleagues reported on 74 metastatic GEP-NETs patients treated with PRRT, observing a PFS and OS of, respectively, 26 and 55 months [4]. In their protocol, short-acting somatostatin analogues needed to be paused for 1 day before the administration of $\left[{ }^{177} \mathrm{Lu}\right]$ Octreotate, while for long-acting analogues, the suspension lasted for a minimum of 4 weeks before PRRT [4].

Furthermore, a better efficacy of $\left[{ }^{177} \mathrm{Lu}\right]$ DOTATATE plus Octreotide-LAR (30 mg) vs. Ocreotide-LAR $(60 \mathrm{mg})$ alone in the treatment of metastatic, progressive, and SSTRspositive GEP-NETs has been demonstrated by the Netter-1 study [5].

In the $\left[{ }^{177} \mathrm{Lu}\right]$ DOTATATE therapy group, the patients continued receiving SSAs approximately $24 \mathrm{~h}$ after each $\left[{ }^{177} \mathrm{Lu}\right]$ DOTATATE cycle, then monthly after fulfilment of all four treatments [5]. Crucially, the study reported a median PFS of 28.4 months in a combined treatment group vs. 8.5 of a control group (follow-up period of about 40 months), while the median OS in the combined treatment group was not reached.

The effectiveness of the combined SSAs plus PRRT therapy was also confirmed in a 2017 study [6], addressing the survival rate of 79 patients with metastatic NET and positive SSTRs imaging. In addition, the SSAs' administration was delivered 1 month after the last cycle of PRRT and then maintained during the follow-up, resulting in an OS of 55 months, a PFS of 39 months, and an event-free survival of 33 months.

As a further development, a recent article by Yordanova [7] highlighted for the first time the concept of PRRT plus SSAs as "Combined and Maintenance Therapy". Here, the patients achieved a 91 months OS when treated with PRRT plus SSAs as a combination and/or maintenance therapy; the median follow-up period was 43.5 months. In contrast, those treated with PRRT had only 47 months of OS. These 
results reached high statistical significance $(p<0.001)$. Furthermore, results on PFS and death rate events were also better in the patients group treated with PRRT plus SSAs both as combined and maintenance therapy.

These results prove the principle that a more effective anti-tumour activity can be achieved with a combined treatment of PRRT plus SSAs, rather than by SSAs only [3-7]. Whilst the above evidence is undoubtedly remarkable and encouraging, a question that still needs to be answered is how one can establish an optimal time schedule of PRRT and SSAs as combined and maintenance therapy.

A procedure similar to that of Yordanova [7], although with a different timing of the SSAs therapy with respect to PRRT, has been tested: the SSAs' LAR treatment was paused for 2 weeks before PRRT [8], instead of 4-6 weeks [7]. The patients were then eligible for another SSAs administration 2 weeks after PRRT, namely for two SSAs injections within the period separating two PRRTs. Finally, after the conclusion of PRRT, the SSAs were administered as a maintenance treatment for all the GEP-NETs patients (Fig. 1). This constitutes a novelty supported by literature data and by a favourable radiopharmaceutical biodistribution imaging (Fig. 2).

The procedure is based on the assumption that the two therapeutic procedures do not interfere negatively, i.e., first, no eventual saturation of SSTRs by SSAs, both unlabelled and labelled; second, no influence of the SSAs therapy on the uptake of the DOTATATE radiopharmaceutical, in both diagnostic and therapeutic models.

The SSTRs kinetics after an SSAs' injection is well understood through experimental in vivo models: the binding of SSAs by SSTRs, highly expressed by NETs cells, induces a very fast internalization of the ligand-receptor complex within $2.5 \mathrm{~min}$. In addition, the recycling process of internalized SSTRs is reversible, and the receptors are found again at the cell surface within $24 \mathrm{~h}$ [9]. This means that a continuous recycling of receptors seems to occur on the NETs' cell membrane.

Accordingly, to reduce the hypothetical interfering effect, the joint EANM-SNNMI-IAEA PRRT-procedure recommends a time interval of 4 weeks between SSAs and $\left[{ }^{177} \mathrm{Lu}\right]$ DOTApeptide administrations. At the same time, the guideline acknowledges that the effects of SSAs' administration with respect to PRRT are yet to be characterized properly [1]. Moreover, a blocking effect in tumour tissue SSTRs could be appreciated after the administration of short-acting SSAs at a dose of 250 or $500 \mu \mathrm{g}$ [10].

However, another recent study comparing the pharmacokinetics of several SSAs formulations (either long-acting or prolonged-release) found that the pharmacokinetic profile of the SSAs formulations demonstrated a blood $\mathrm{C}_{\max }$ and $\mathrm{C}_{\text {mean }}$ of the order of nanogram/millilitre 14 days after the administration [11].

According to the LUTATHERA ${ }^{\circledR}$ product characteristics, the labelled peptide oxodotreotide amounts to about $10 \mu \mathrm{g} /$ $\mathrm{mL}$ [12]. At such a low dosage, the SSAs do not exert any clinically relevant pharmacodynamic effect; rather, they simply direct the $\left[{ }^{177} \mathrm{Lu}\right]$ DOTApeptides therapy to the SSTRs expressed by NETs' cancer cells.

In a previous study, in which an amount of radiolabelled peptide similar to that of the LUTATHERA ${ }^{\circledR}$ dosage was used [8], there was no interference with the saturation of tumour SSTRs when the long-acting or prolonged-release SSAs administration was suspended 2 weeks before the PRRT. A post-therapy whole-body scan performed in all

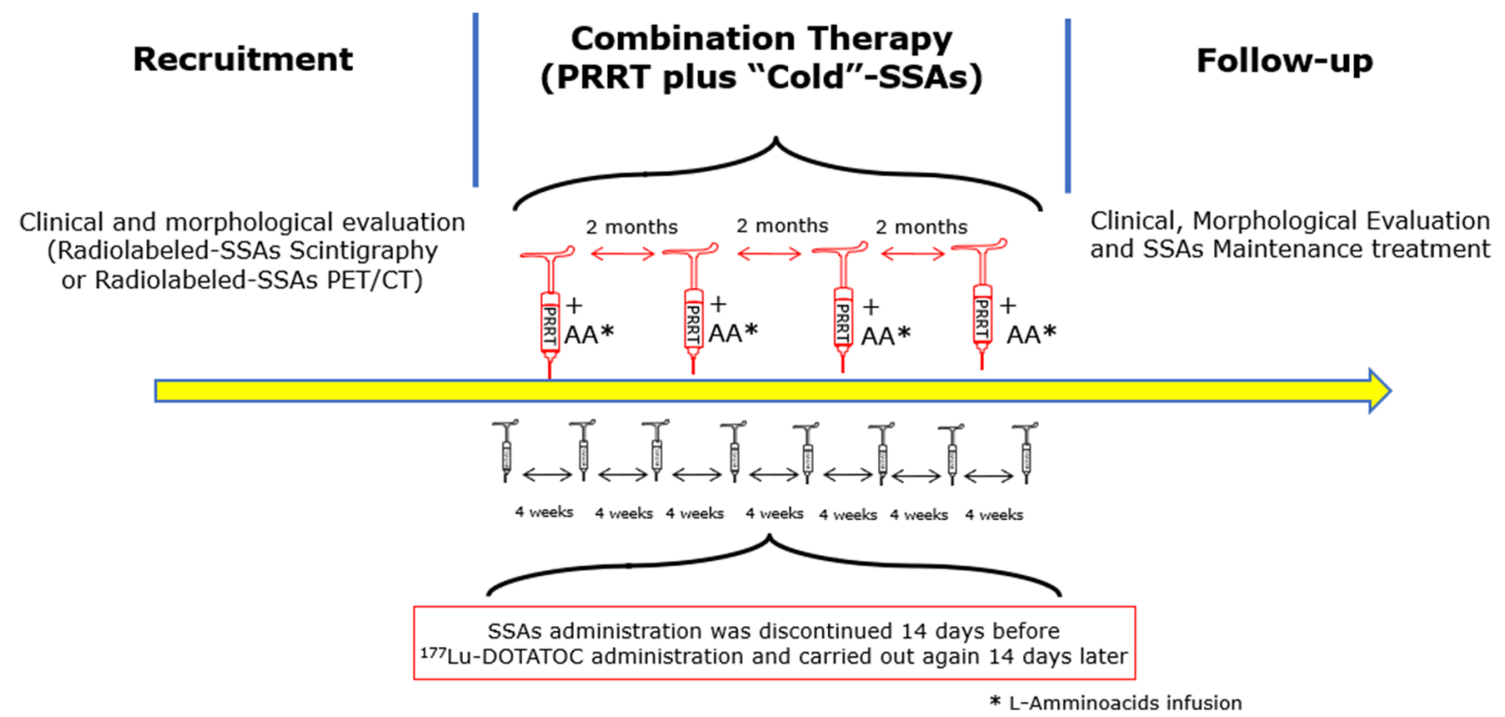

Fig. 1 Proposed withdrawal schedule for SSAs and PRRT 


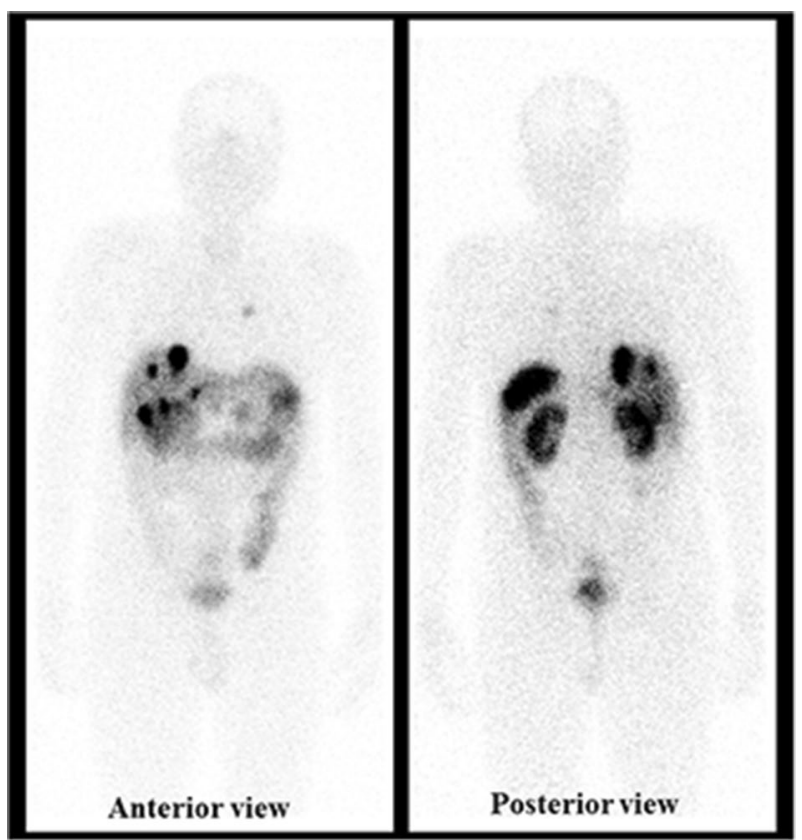

Fig. 2 p-NET male patient with metastatic lymph nodes and liver metastases. The post-therapy whole-body scan was performed approximately $24 \mathrm{~h}$ after PRRT

patients confirms indeed a favourable radiopharmaceutical biodistribution (Fig. 2).

The second point one should address is a possible influence of the SSAs' therapy on the uptake of DOTATATE radiopharmaceutical. Several authors have demonstrated very clearly the benefits of a radiopharmaceutical-DOTApeptide administration when used in conjunction with SSAs (short- or long-acting formulation) [10, 13, 14]. Regarding the diagnostic procedure, a prior SSAs' administration seems to produce a better image contrast between target lesions and normal tissue, together with a "tumour sink phenomenon" [15] that decreases the $\left[{ }^{177} \mathrm{Lu}\right]$ DOTApeptide uptake in healthy organs.

Velikan et al. demonstrated indeed that injecting $50 \mu \mathrm{g}$ of octreotide 10 min before $\left[{ }^{68} \mathrm{Ga}\right]$ DOTATOC produced an uptake that increased in the metastases but decreased in the normal tissues (liver and spleen) [10]. Similar results were detected for the long-acting and prolonged-release SSAs.

In this regard, Cherk and colleagues proved that a chronic long-acting SSAs administration prior to $\left[{ }^{68} \mathrm{Ga}\right]$ DOTApeptide imaging may improve the binding of the latter at the disease sites, also protecting the critical organs as a welcome additional effect [13].

Aalbersberg et al. have recently assessed the influence of Lanreotide administration on the $\left[{ }^{68} \mathrm{Ga}\right]$ DOTATATE uptake in advanced NET patients, who were treated the previous day with prolonged-release SSAs [14]. In their prospective data, the SSAs injection does not interfere in the $\left[{ }^{68} \mathrm{Ga}\right]$
DOTApeptide-SSTRs binding. On the contrary, it positively affects the uptake of the lesion (increased uptake) as well as that of target organs such as thyroid, spleen, and liver (decreased uptake) [14].

The explanation of this phenomenon is likely based on the differences in internalization patterns in metastatic cells vs. normal tissue cells, together with the potential upregulation of the SSTRs expression of metastatic cells after SSAs' administration [16]. Moreover, the literature evidence seems to suggest a potential "Rituximab effect", namely the saturation of SSAs receptors also in healthy tissues [13]. It was hypothesized indeed that the administration of LA-SSA therapy prior to PRRT may improve the PRRT anti-tumour activity in most patients, by saturating the physiological binding sites and by increasing the binding of $\left[{ }^{177} \mathrm{Lu}\right]$ Dotapeptide-SSTRs in pathological sites, just like the administration of 'cold' Rituximab prior to administration of radiolabelled Rituximab in patients with CD20 + B-cell lymphoma [13].

The non-interference of SSAs (either short- or long-acting) represents an advantage also for the patient, since it is not necessary to suspend the therapy for a long time, and is beneficial for the scheduling of admissions as well [14].

A study in a cohort of 40 metastatic and advanced GEPNETs patients enrolled for PRRT and subjected to this procedure has provided some preliminary results. The clinical data show that OS and PFS did not reach the median value. [8]. Moreover, the treatment has proven to be safe and effective: all patients had stable/improved carcinoid syndrome symptoms; few toxicities were observed; no safety issues were identified [8].

The above findings clearly show the necessity of establishing the most effective and beneficial time interval between SSAs and $\left[{ }^{177} \mathrm{Lu}\right.$ ]DOTApeptides administrations.

To summarize, it was found in the NETTER-1 trials [5] and in a preliminary study [8] that the withdrawal of SSAs therapy with respect to PRRT may be reduced to 2 weeks without altering the efficacy of both $\left[{ }^{177} \mathrm{Lu}\right]$ therapy and SSAs. At the same time, this approach allows also the use of two complementary treatments by exploiting their special synergies.

Additional investigations and clinical trials with larger number of patients are clearly needed. Interesting developments, however, could follow from the favourable half-life (6,65 days) of $\left[{ }^{177} \mathrm{Lu}\right]$ DOTATATE, since the gamma-ray emission would allow subsequent sequential quantitative SPECT/CT scans aimed at targeting the main lesions. This would provide both a further understanding of the SSRs' distribution pattern in the metastatic areas and of the pharmacokinetic profile of $\left[{ }^{177} \mathrm{Lu}\right] \mathrm{DOTApeptides}$ in the lesions. 
Author contributions SAP: literature search and review, study concepts, study design, and manuscript writing. AF: literature search and review, content planning, manuscript writing, and editing. SB: study concepts, study design, and manuscript review.

Funding None.

\section{Compliance with ethical standards}

Conflict of interest The authors declare that they have no conflicts of interest.

Ethical statement This article does not contain any studies involving animals performed by any of the authors.

Ethical approval All procedures performed in this study were in accordance with the ethical standards of the institutional research committee and the national regulations and also with the principles of the 1964 Declaration of Helsinki and its later amendments as far as they are required for this type of study.

\section{References}

1. Bodei L, Mueller-Brand J, Baum RP et al (2013) The joint IAEA, EANM, and SNMMI practical guidance on peptide receptor radionuclide therapy (PRRNT) in neuroendocrine tumours. Eur J Nucl Med Mol Imaging 40(5):800-816

2. Pokuri VK, Fong MK, Iyer R (2016) Octreotide and lanreotide in gastroenteropancreatic neuroendocrine tumors. Curr Oncol Rep. 18(1):7

3. Brabander T, van der Zwan WA, Teunissen JJM et al (2017) Long-term efficacy, survival, and safety of [177Lu-DOTA0, Tyr3] octreotate in patients with gastroenteropancreatic and bronchial neuroendocrine tumours. Clin Cancer Res 23(16):4617-4624

4. Ezziddin S, Khalaf F, Vanezi M et al (2014) Outcome of peptide receptor radionuclide therapy with $177 \mathrm{Lu}$-octreotate in advanced grade 1/2 pancreatic neuroendocrine tumours. Eur J Nucl Med Mol Imaging 41(5):925-933

5. Strosberg J, El-Haddad G, Wolin E et al (2017) Phase 3 Trial of 177Lu-Dotatate for midgut neuroendocrine tumours. N Engl J Med 376(2):125-135
6. Sowa-Staszczak A, Stefanska A, Chrapczynski P et al (2017) Does combination of "cold" and "hot" somatostatin analogs prolong survival of patients with neuroendocrine neoplasms? Endocr J 64(2):171-177

7. Yordanova A, Wicharz MM, Mayer K et al (2018) The role of adding somatostatin analogues to peptide receptor radionuclide therapy as a combination and maintenance therapy. Clin Cancer Res 24(19):4672-4679

8. Pignata SA, Sturiale L, Laudicella R et al (2019) Peptide receptor radionuclide therapy and somatostatin "cold" analogues therapy in the treatment of GEP-NET patients: our center' experience. Clin Transl Imaging 7(Suppl 1):S56

9. Waser B, Tamma ML, Cescato R et al (2009) Highly efficient in vivo agonist-induced internalization of sst 2 receptors in somatostatin target tissues. J Nucl Med 50(6):936-941

10. Velikyan I, Sundin A, Eriksson B et al (2010) In vivo binding of [68 Ga]-DOTATOC to somatostatin receptors in neuroendocrine tumours-impact of peptide mass. Nucl Med Biol 37(3):265-275

11. Astruc B, Marbach P, Bouterfa $\mathrm{H}$ et al (2005) Long-acting octreotide and prolonged-release lanreotide formulations have different pharmacokinetic profiles. J Clin Pharmacol 45(7):836-844

12. LUTATHERA ${ }^{\circledR}$ Summary of product characteristics. http://www. ema.europa.eu

13. Cherk MH, Kong G, Hicks RJ et al (2018) Changes in biodistribution on 68 Ga-DOTA-Octreotate PET/CT after long acting somatostatin analogue therapy in neuroendocrine tumour patients may result in pseudoprogression. Cancer Imaging 18(1):3

14. Aalbersberg EA, de Wit-van der Veen BJ, Versleijen MWJ et al (2019) Influence of lanreotide on uptake of 68 Ga-DOTATATE in patients with neuroendocrine tumours: a prospective intra-patient evaluation. Eur J Nucl Med Mol Imaging 46(3):696-703

15. Beauregard JM, Hofman MS, Kong G et al (2012) The tumour sink effect on the biodistribution of 68 Ga-DOTA-octreotate: implications for peptide receptor radionuclide therapy. Eur J Nucl Med Mol Imaging 39(1):50-56

16. Kuyumcu S, Özkan ZG, Sanli Y et al (2013) Physiological and tumoral uptake of (68)Ga-DOTATATE: standardized uptake values and challenges in interpretation. Ann Nucl Med 27(6):538-545

Publisher's Note Springer Nature remains neutral with regard to jurisdictional claims in published maps and institutional affiliations. 